\title{
Models Comparison And Validation With the Experimental Nano- Scale Silicon Substrate Based Annealed Pt/HfO2/Pt Resistive Random Access Memory Cell
}

Napolean A ( $\sim$ nepojustin@gmail.com )

Karunya Institute of Technology and Sciences https://orcid.org/0000-0001-8421-2454

\section{Sivamangai NM}

Karunya Institute of technology and Science: Karunya Institute of Technology and Sciences

Nithya N

Kumaraguru College of Technology

Naveenkumar $\mathbf{R}$

Karunya Institute of technology and Science: Karunya Institute of Technology and Sciences

\section{Research Article}

Keywords: RRAM, Annealing, Modeling and simulation, Non-linear ion drift, Yakopcic, VTEAM models

Posted Date: July 14th, 2021

DOI: https://doi.org/10.21203/rs.3.rs-704249/v1

License: (c) (1) This work is licensed under a Creative Commons Attribution 4.0 International License.

Read Full License 


\title{
Models Comparison And Validation With the Experimental Nano-
}

\section{Scale Silicon Substrate Based Annealed Pt/HfO $/ \mathrm{Pt}$ Resistive Random}

\section{Access Memory Cell}

* Napolean A, Karunya Institute of Technology and Sciences Coimbatore, India, nepojustin@gmail.com

Sivamangai NM, Karunya Institute of Technology and Sciences Coimbatore, India, nmsivam@gmail.com

Nithya N, Kumaraguru College of Technology, Coimbatore, India, nithyaame@gmail.com

Naveenkumar R, Karunya Institute of Technology and sciences Coimbatore, India, naveentamil256@gmail.com

* Corresponding Author

\begin{abstract}
Metal oxide resistive random access memory (RRAM) is a novel device that provides an alternate solution for existing CMOS memory devices. In RRAM, correlate the experimental result with a simulated result by a unique model is a critical task. This work focused on the validation of silicon substrate-based fabricated single layer annealed and electroformed at $8^{\circ} \mathrm{C}$ ambient temperature (A-80) RRAM cell. The experimental result concludes that the proposed $\mathrm{Pt} / \mathrm{HfO}_{2} / \mathrm{Pt}$ device provides the forming voltage of $3.8 \mathrm{~V}$, Vset $=1.7 \mathrm{~V}$, and Vreset=-0.8 $\mathrm{V}$. Switching results are compared with the simulated result which is working based on non-linear ion drift, Yakopcic and voltage threshold adaptive memristor (VTEAM) models. VTEAM model gives a closure relationship with experimental data and well suited for our fabricated device. Further, the VTEAM model is modified to contribute accurate results and one of the standard model metrics, accuracy is analysed for a modified VTEAM model. Statistical analysis proves that, the mean error percentage of modified VTEAM and VTEAM models against experimental outcomes are $21.4 \%$ and $25.3 \%$ respectively

Keywords: RRAM, Annealing, Modeling and simulation, Non-linear ion drift, Yakopcic, VTEAM models
\end{abstract}

\section{INTRODUCTION}

RRAM is the most capable non-volatile storage device owing to its quality of nanoscale, simple structure, costeffectiveness, quick switching speed, and great compatibility with the CMOS procedure. Among many types of RRAM, metal oxide RRAM is a unique characteristic of matured fabrication compatibility with the CMOS process [1-3]. The bottleneck in RRAM is maintaining switching uniformity for entire periods. We rectify this challenge by device dimensions with stack layer structure [4]. The reduction of operating voltage in a scaled RRAM is a major constraint. Post-metal annealing (PMA) is an effective fabrication process to mitigate the voltage reduction problem. [5]. Developing a unique model for a nanoscale metal oxide RRAM device is an important necessity to check its behaviour in a device, circuit-level, and for system implementation. The complicated physics in RRAM leads to 
confusion to generate the generalized model for a new device. In literature, many models are studied and verified. Still, no universal model is recommended for an RRAM device. Among that, researchers are more interested in few models (non-linear ion drift, Yakopcic, and VTEAM) which are supported to bipolar, voltage-controlled with the state variables of the doped and undoped region. [6].

The motivation of this work is to fabricate a single layer metal oxide $\mathrm{Pt} / \mathrm{HfO}_{2} / \mathrm{Pt} \mathrm{RRAM}$ cell and the experimental switching results are measured. Fabricated results are compared with a simulation result based on the three different models. We organize this paper: Section 2 highlights the fabrication results of the device. Section 3 presents the three different models and the modified VTEAM models for the proposed device structure. Section 4 demonstrates the simulated results of three different models with a modified models in cadence environment and the comparison of the switching result of the fabricated device with all the different model cadence output. In section 5 the important model metric is analysed for the optimized VTEAM model.

\section{FABRICATION RESULTS}

Single layer metal oxide nanoscale annealed $\mathrm{Pt} / \mathrm{HfO}_{2} / \mathrm{Pt} \mathrm{RRAM}$ device is fabricated by sputtering, atomic layer deposition and annealing processes on the RCA cleaned and wet oxidised silicon substrate. Schematic structure, fabrication process flow, FIB-SEM cross-sectional view, XRD analysis, forming voltage $\left(\mathrm{V}_{\mathrm{f}}\right)$, and $\mathrm{I}-\mathrm{V}$ characteristics are shown in Figures $1 \mathrm{a}, 1 \mathrm{~b}, 1 \mathrm{c}, 1 \mathrm{~d}, 1 \mathrm{e}$ and $1 \mathrm{f}$. Results confirmed the low $\mathrm{V}_{\mathrm{f}}$ and better memory switching function. The effect of annealing yields less operating voltage and better reliability.

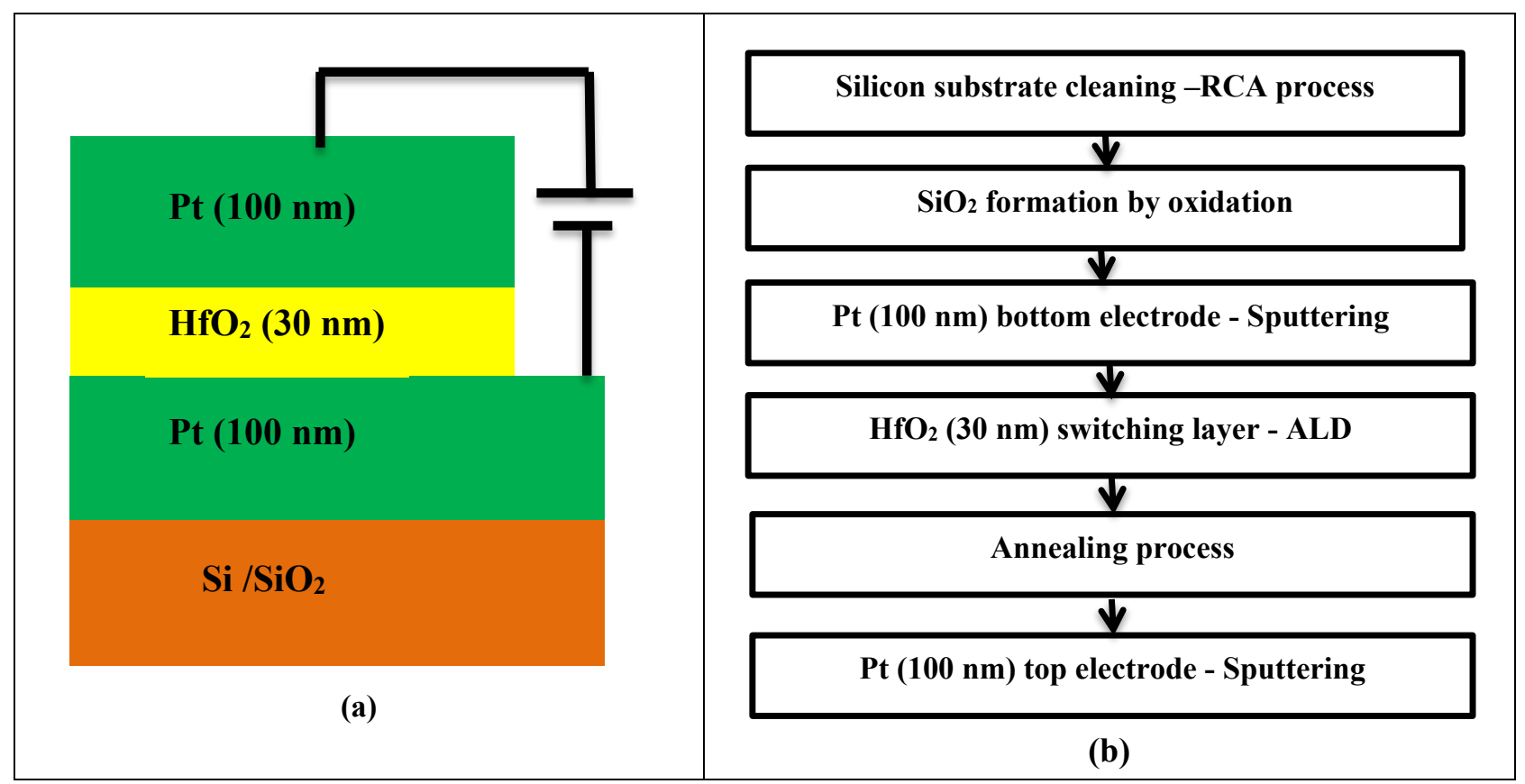




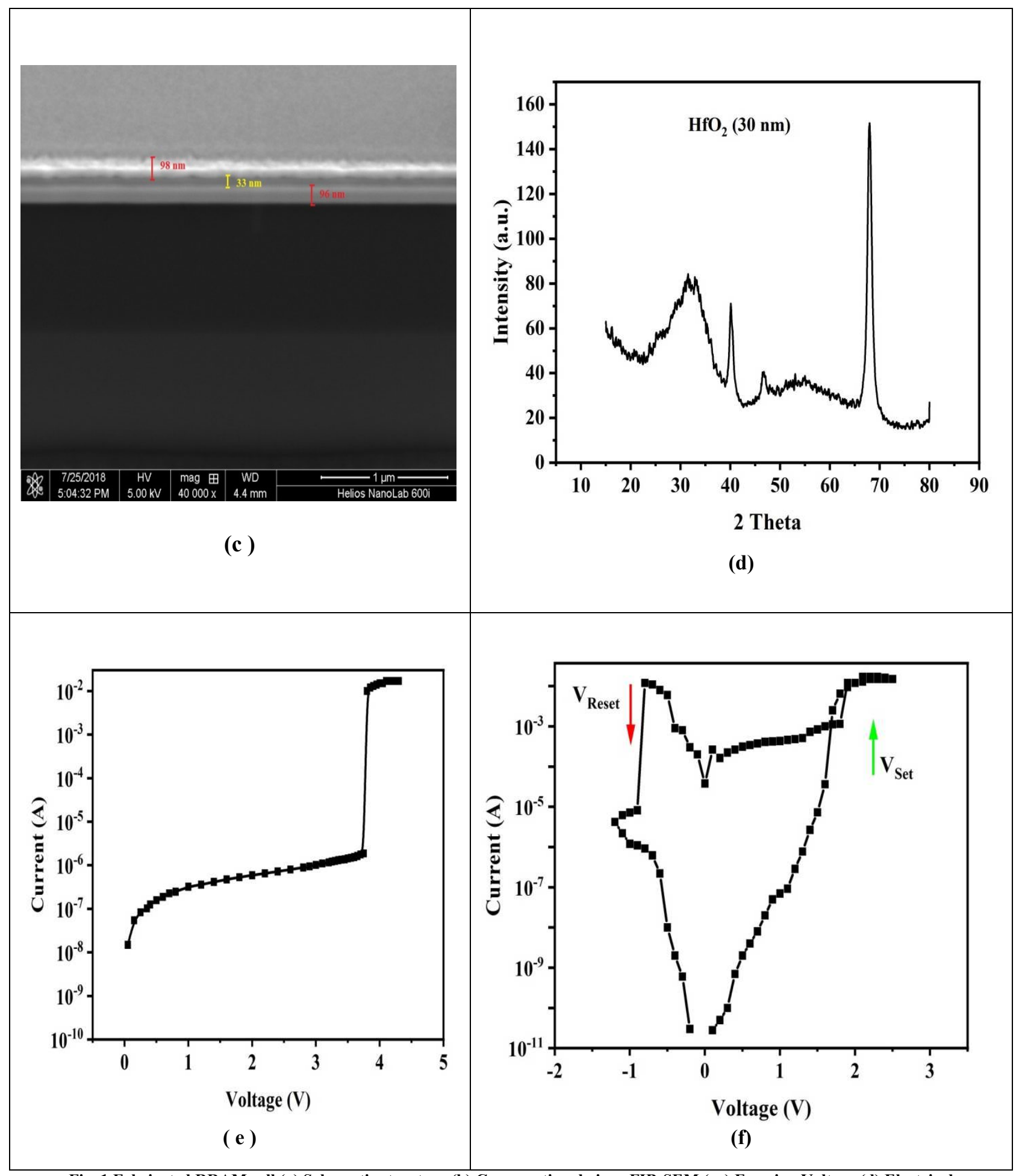

Fig. 1 Fabricated RRAM cell (a) Schematic structure (b) Cross sectional view- FIB-SEM ( c ) Forming Voltage (d) Electrical characteristics

After annealing the total number of oxygen vacancy is improved considerably. It leads to the quick creation of conducting filament in the switching layer. Fig $1 \mathrm{c}$ shows the $\mathrm{V}_{\mathrm{f}}$ value of a annealed device (A-80). The annealed device gives a reduced $\mathrm{V}_{\mathrm{f}}$ value of $3.8 \mathrm{~V}$. Fig $1 \mathrm{~d}$ display the $\mathrm{DC}$ bipolar switching analysis of the fabricated device. This 
RRAM cell sample gives a very low Vset $=1.7 \mathrm{~V}$ and Vreset $=-0.8 \mathrm{~V}$. This result is comparable with an annealed tri layer structure [7].

\section{MODELS SELECTION}

Validating the proposed device experimental result is mandatory for a successful implementation at a circuit level. Generating a unique model for RRAM is a complexity. In this paper, the experimental results are validated with three general models as per our optimized structure. Since the top switching layer is annealed, it creates number of oxygen vacancies in between the top $\mathrm{Pt}$ and $\mathrm{HfO}_{2}$ layer. This effect leads the device structure state variable are doped and undoped region and gives the bipolar switching operation is present in fig 2.

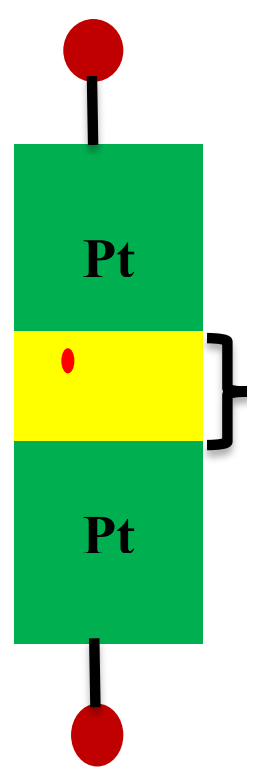

(a) Initial State

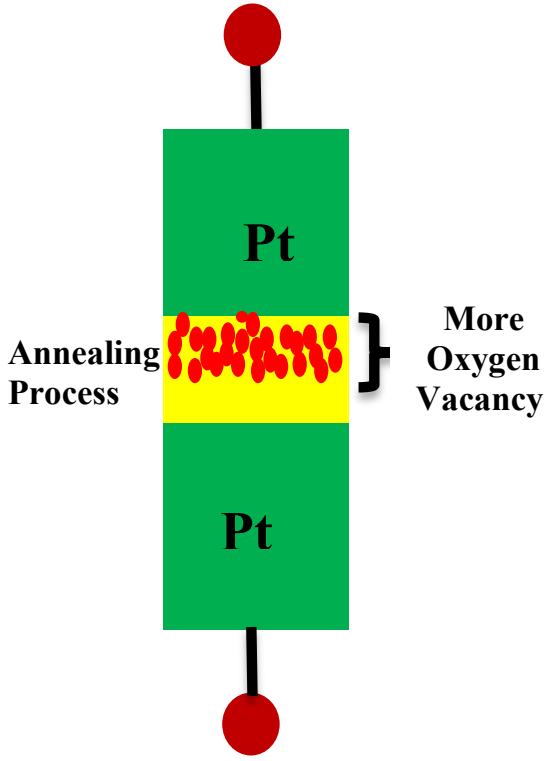

(b) After Annealing

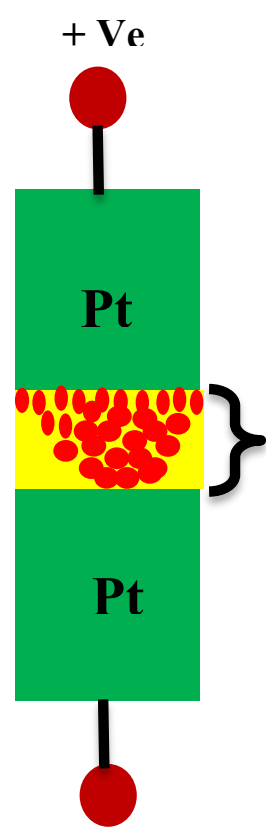

$-\mathrm{Ve}$

(c) Set State

- Ve

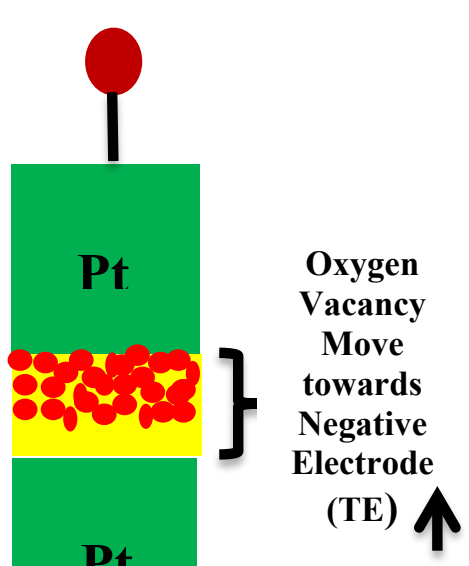

Fig. 2 Bipolar switching operation based on oxygen vacancy

Moreover, the constructed device results prove the memory cell type is a bipolar with voltage-controlled mechanism. Suitable models are selected to satisfy these above-mentioned conditions. Finally, among the various models [6] Non-linear ion, Yakopcic and VTEAM models are selected for the validation purpose.

\subsection{Non-linear ion drift model for the proposed structure}


The non-linear behaviour of RRAM that is fabricated leads to the development of a non-linear ion drift model. This can be observed from the various experimental results [8]. It is a bipolar device controlled by voltage. It is a non-linear and non-symmetric device with high complexity. Spatial heterogeneous metal/oxide electronic barrier controls the conduction in RRAM devices [9]. The movement of positively charged oxygen vacancies contribute a d ynamic switching. This oxygen vacancies functioning as original dopants will establish or discontinue the filament path that conduct over this electronic blockade. At the boundaries, metal/oxide coherences oxygen vacancy concentration is higher. The active electrode which is a top electrode of the MIM structure is the place where dynamic ON and OFF switching will take place. The resultant resistance of the memristor $\mathrm{R}_{\mathrm{MEM}}$ is the addition of doped and undoped area

$$
R_{M E M}(x)=R_{O N}(x)+R_{O F F}(1-\mathrm{x})
$$

Memristors worked as dynamic resistors. Dynamic resistors are the ones that can vary its state in line with the time integral of the incident voltage or current. Yang et al. were could not specify a relationship on dynamic state variables. The I-V relation is explained as

$$
I=w^{n} \beta \sinh (\alpha \mathrm{V})+\chi\left(\exp \left(\gamma^{V}\right)-1\right)
$$

$\beta, \gamma, \chi$ and $\eta$ are termed to be as fitting constants. The ON state of the memristor is approached by the term $\beta \sinh (\alpha \mathrm{V})$ in which the electron passes the thin residual electronic barrier. The device state variable (w) range is fixed from 0 $(\mathrm{ON})$ to $1(\mathrm{OFF})$. In equation (2) the second term denotes the memristor OFF state and the term $\eta$ is known as free parameter that is helped to change the switching states. The non-linear drift effects are defined by certain window functions that define the leap for the device. HP team [10] was proposed that multiplication of window function with the state variable is given by

$$
f(x)=w(1-w) / D^{2}
$$

For the I-V curve modelling of the manufactured device, using the above equation the best fit is obtained, when $14 \leq$ $n \leq 22$. It made clear that the resultant dopant drift rate is very non-linearly dependent on the voltage applied to the device. Therefore, this drift phenomenon at the interfaces is accepted as nonlinear. Lehtonen and Laiho proposed a new relationship for the state variable $\mathrm{w}$ and is implemented in SPICE $[11,12]$. This model is given as [10]

$$
\frac{d w}{d t}=a \times f(w) \times g(v)
$$

Here ' $\mathrm{a}$ ' is the constant, $\mathrm{R}$ is the real number, $\mathrm{f}[0,1] \rightarrow R$ reviewed as a window function and $g: R \rightarrow R$ is a linear function which is considered in the basic linear model. $\mathrm{g}(\mathrm{v})$ is a nonlinear, odd and monotonically increasing function. The proposed nonlinear equation is

$$
g(v)=v^{q}
$$


The switching between ON and OFF state is very quick in RRAM models. Here, the switching speed is depending on the exponent term q. This model is implemented in a SPICE environment for high switching speed circuits [11, 12]. In SPICE, the model is verified, whether the particular model is suitable for implementation or cannot be implemented in the circuit. Although many improvements were made to the later model, it was precise because of its high accuracy that this model became the basis for the remaining RRAM models.

\subsection{Yakopcic model}

The bipolar voltage-controlled device model was originally tested for TiO2 systems. Although the yakopcic model [13, 14] was not specifically validated for RRAM cell at its initial period, it resembled numerous RRAM fabricated memory cells. Pickett -Adbala model $[15,16]$ is a source of the yakopcic model, which uses similar state variables but has been altered to incorporate with a synaptic function for neuromorphic system applications. The model has been tested to use in practical neuromorphic systems.

This model's state variable $\mathrm{w}(\mathrm{t})$ is defined with the boundary condition of 0 and 1 . Model equation for the current is given as,

$$
I(t)= \begin{cases}a_{1} w(t) \sinh (b v(t)), & v(t) \geq 0 \\ a_{2} w(t) \sinh (b v(t)), & v(t)<0\end{cases}
$$

State variables switching is controlled by $g(v(t))$ and $f(x(t))$. Moreover, the state variable alteration is dominated by the threshold voltage. When the input voltage is reached greater/lesser than its threshold voltage, the device structure is changing. The term $g(v(t))$ function simulates the device turn-on and turnoff conditions, taking input voltage polarity into account. When the voltage set $\left(\mathrm{v}_{\mathrm{p}}\right)$ and reset $\left(\mathrm{v}_{\mathrm{n}}\right)$ have different threshold values, this better agrees with the bipolar practical devices. This can be expressed as

$$
g(v(t))=\left\{\begin{aligned}
A_{p}\left(e^{v(t)}-e^{v_{p}}\right), & v(t)>v_{p} \\
-A_{n}\left(e^{-v(t)}-e^{v_{n}}\right), & v(t)<-v_{n} \\
0, & -v_{n} \leq v(t) \leq v_{p}
\end{aligned}\right.
$$

$A_{p}$ and $A_{n}$ controls the speed of filament formation/rupture, these parameter depends on the type of dielectric material which is selected in a switching layer. Threshold support is built into the model to improve applicability. The change of the state variable modelled by $\mathrm{f}(\mathrm{w}(\mathrm{t}))$ limits the variable. Vp and vn are stand for threshold voltages. These values are based on the amount of oxygen vacancies in a memristor at its initial stage. High quantity of oxygen vacancy leads to reduced threshold voltages. It is basically like a window function, restricting state change variables to certain limits, such as

$$
f_{p}\left(w, w_{p}\right)=\frac{w_{p}-w}{1-w_{p}}+1
$$




$$
f_{n}\left(w, w_{n}\right)=\frac{w}{1-w_{n}}
$$

As the model was modelled using HP Labs [8]. Data on the characteristics of the TiO2 memristor, the fitting of the device is good only when the calibration of fitting parameters are properly done. To understand the degree to which the system can withstand variability, the variability must be analysed. If the critical point is reached, read/write errors may occur in the device. The equation of the function $G(V(t))$ that describes the device threshold is slightly modified accordingly as

$$
G(V(t))=\frac{V}{R_{\text {on }}}+\frac{(1-V)}{R_{\text {off }}}
$$

Where $R_{o n}$ is the minimum device resistance and Roff is the maximum device resistance. The values applied are based on the values extracted from the fabricated device.

\subsection{VTEAM Model}

This model combines the advantage of a TEAM model. It focuses the threshold voltage value rather than threshold current [17]. Mathematically,

$$
\begin{aligned}
& \frac{d w}{d t}=f(w, v) \\
& i(t)=G(w, v) \cdot v(t)
\end{aligned}
$$

Where $\mathrm{w}$ is a state variable, $\mathrm{v}(\mathrm{t})$ is a voltage over the device, $\mathrm{i}(\mathrm{t})$ - current through that memristor, $\mathrm{G}(\mathrm{w}, \mathrm{v})$ is a conductance of a cell, and $\mathrm{t}$ is a time factor. State variable derivative is given by

$$
\frac{d w(t)}{d t}=\left\{\begin{array}{c}
k_{o f f} \cdot\left(\frac{v(t)}{v_{o n}}-1\right)^{\alpha_{o f f}} \cdot f_{o f f(w)}, 0<v_{o f f}<v \\
0, \quad v_{o n}<v<v_{o f f} \\
k_{\text {on }} \cdot\left(\frac{v(t)}{v_{o n}}-1\right)^{\alpha_{o n}} \cdot f_{\text {on }(w)}, v<v_{\text {on }}<0
\end{array}\right.
$$

Where $\mathrm{k}_{\mathrm{off}}, \mathrm{k}_{\mathrm{on}}, \alpha_{\mathrm{off}}$, and $\alpha_{\mathrm{on}}$ are constants, $\mathrm{v}_{\mathrm{on}}-$ Threshold on voltage, $\mathrm{v}_{\mathrm{off}}-$ Threshold off. Parameter $\mathrm{k}_{\mathrm{off}}$ and $\mathrm{k}_{\mathrm{on}}$ are positive and negative numbers, foff $(\mathrm{w})$ and $\mathrm{f}_{\mathrm{on}}(\mathrm{w})$ denotes the derivative off and on state variable w. The window function is $\mathrm{w} \in\left[\mathrm{w}_{\mathrm{on}}, \mathrm{w}_{\mathrm{off}}\right]$. The relationship between current and voltage with the state variable is

$$
i(t)=\left[R_{o n}+\frac{R_{o f f}-R_{o n}}{w_{o f f}-w_{o n}} \cdot\left(w-w_{o n}\right)\right]^{-1} \cdot v(t)
$$

Where $\mathrm{w}_{\mathrm{on}}$ and $\mathrm{w}_{\text {off }}$ denotes the boundary of internal state variable $\mathrm{w} ; \mathrm{R}_{\mathrm{on}}$ and $\mathrm{R}_{\text {off }}$ represents the corresponding resistance values of the device. Previous equation can be modified in terms of exponentially relating with the state variable is

$$
i(t)=\frac{e^{-\frac{\lambda}{w_{o f f-w_{o n}}} \cdot\left(w-w_{o n}\right)}}{R_{o n}} \cdot v(t)
$$


Where $\lambda$ is a fitting parameter, and $e^{\lambda}=\frac{R_{\text {off }}}{R_{\text {on }}}$.

\subsubsection{Modified VTEAM model}

In general the VTEAM model the electroforming ambient temperature $\left(T_{f}\right)$ is not taken into account as. But this temperature plays a vital role in the RRAM device switching operation [7]. We modified VTEAM model, considering this electroforming environment temperature coefficient $\left(E_{T}\right)$. This value is to be dependent on the $T_{f}$ and reference temperature value $\left(\mathrm{T}_{\mathrm{r}}=298 \mathrm{~K}\right)$. Equation (15) is made different into

$$
\begin{gathered}
i(t)=\frac{e^{-\frac{\lambda}{w_{o f f-w_{o n}}} \cdot\left(w-w_{o n}\right)}}{R_{o n}} \cdot v(t) \cdot\left(E_{T}\right) \\
\left(E_{T}\right)=e\left(\frac{T_{f}-T_{r}}{T_{r}}\right)
\end{gathered}
$$

Equation (16) shows that the current value is exponentially varied with the forming ambient temperature. High $\mathrm{T}_{\mathrm{f}}$ leads to the increased current value; it modulates the $\mathrm{V}_{\text {set }}$ and $\mathrm{V}_{\text {reset }}$ values.

\section{SIMULATED RESULTS COMPARISON}

This section deals with the simulation results got from the cadence environment for an RRAM symbol which is developed with a suitable Verilog A code for non-linear ion drift, Yakopcic and VTEAM models. The simulation setup is created in $90 \mathrm{~nm}$ technology with a peak-to-peak voltage of $2 \mathrm{~V}$ between two electrodes. The source pulse signal frequency is $1 \mathrm{MHz}$ with a $100 \mathrm{~ns}$ period and $1 \mathrm{~ns}$ rise and fall time. We implemented it in a $1 \mathrm{R}$ device as shown in figure 3. We executed simulation with the fixed values for, peak-to-peak voltage, frequency, time period, rise, and fall time. In, all three models the same fixed vales are given as an input.

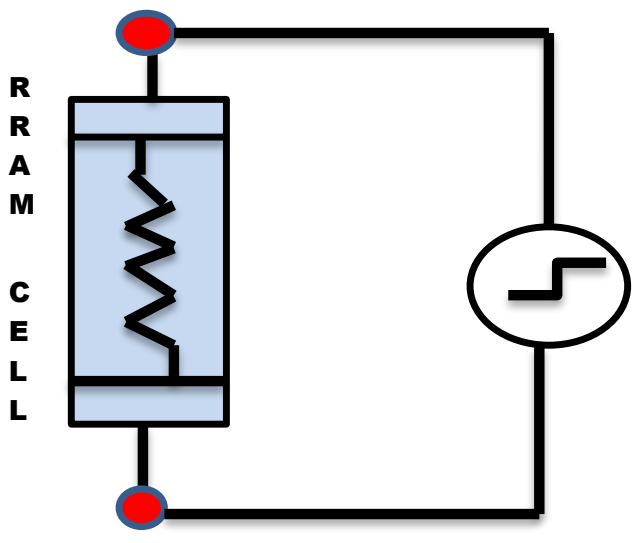

Fig. 3 1R RRAM single Cell

When the top electrode (TE) is connected with a +ve pulse input signal, due to its bipolar switching operation simulated RRAM cell reaches to set (device ON) condition with the voltage of Vset. 


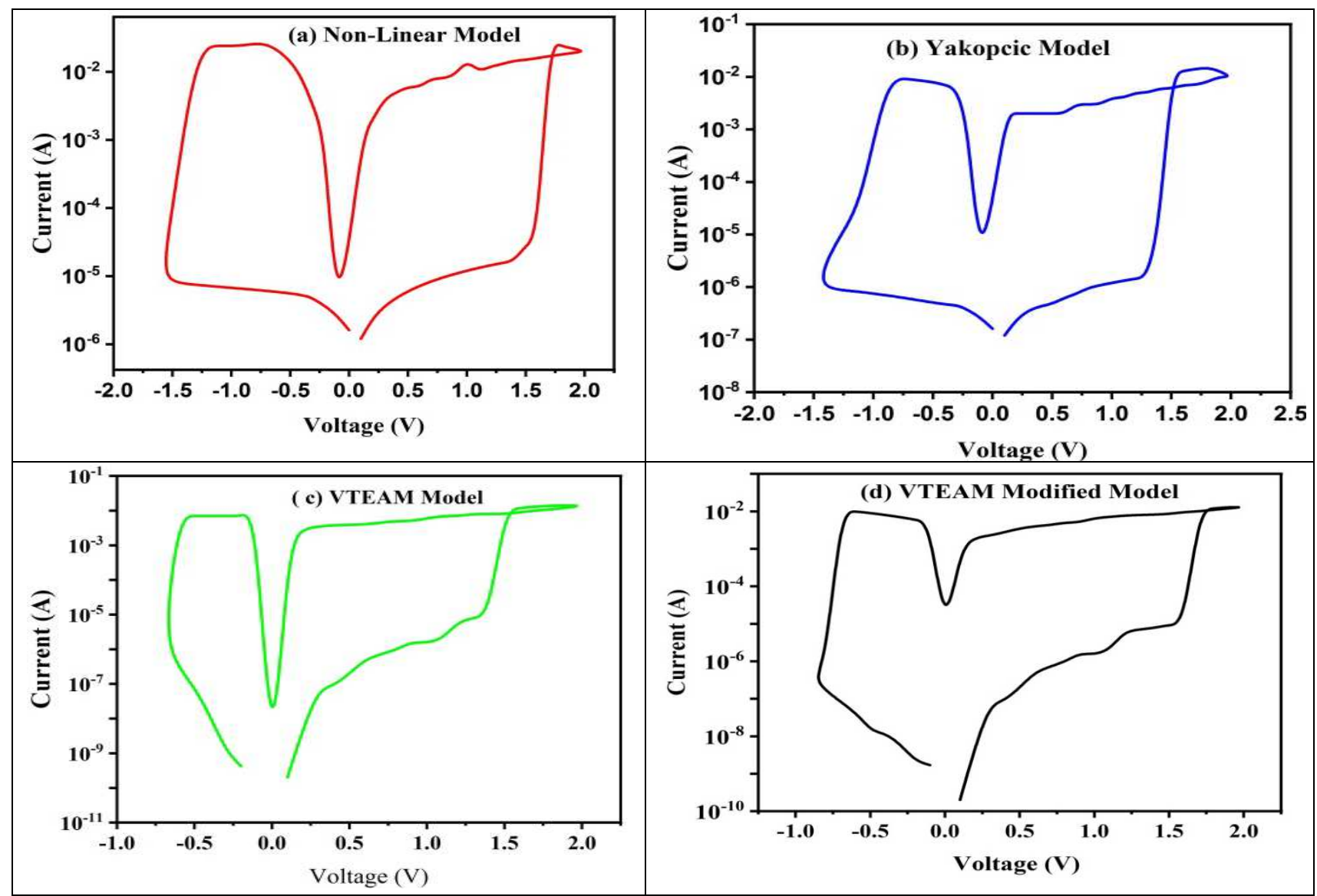

Fig. 4 Simulated I-V response of three different and VTEAM modified models

To reach to set time it took the time (Tset) and the cell resistance value is decreased (Ron). When the -ve pulse is applied on the top electrode the memory cell reach to reset (device OFF) condition with the voltage of Vreset. The time taken to this state is Treset and the corresponding state resistance value is increased (Roff). In figure 4 the basic bipolar I-V characteristics of the simulated RRAM cell for the three models are displayed. Switching parameters are extracted from the simulated figure 5, the corresponding values and experimental data are tabulated in table 1 .

Table.1 Extracted data from experiment and simulation

\begin{tabular}{|c|c|c|c|c|c|c|}
\hline Model & $\begin{array}{c}\text { Vset } \\
(\mathrm{V})\end{array}$ & $\begin{array}{c}\text { Ron } \\
(\mathrm{K} \Omega)\end{array}$ & $\begin{array}{c}\text { Tset } \\
(\mathrm{ns})\end{array}$ & $\begin{array}{c}\text { Vreset } \\
(\mathrm{V})\end{array}$ & $\begin{array}{c}\text { Roff } \\
(\mathrm{K} \Omega)\end{array}$ & $\begin{array}{c}\text { Treset } \\
(\mathrm{ns})\end{array}$ \\
\hline Non-Linear ion & 1.62 & 4.63 & 5.7 & -1.45 & 125 & 6.2 \\
\hline Yakopcic & 1.4 & 9.3 & 6.4 & -1.0 & 624 & 7.7 \\
\hline VTEAM & 1.56 & 7.5 & 8.7 & -0.7 & 501 & 9.3 \\
\hline $\begin{array}{c}\text { VTEAM } \\
\text { Modified }\end{array}$ & 1.72 & 6.8 & 9.3 & -0.84 & 619 & 10.6 \\
\hline Experimental & 1.7 & 6 & 10 & -0.8 & 627 & 12 \\
\hline
\end{tabular}

Results confirm that fabricated device experimental values are closely fit with the VTEAM model compared to other 
models. Also the experimental Vset and Vreset values are exactly comparable with a modified VTEAM model. In a non-linear ion model, the resistance switching traits are nonlinear. Moreover, the simulated output I-V curve depends on the input signal frequency, magnitude of the signal, physical dimension of the active layer and the vacancy mobility. When we increase the input signal frequency, the double hysteresis loop is reduced and reaches its linear state, the memory switching is affected. [10]. Speed of the switching is achieved with high mobility dopants, so it can easily crosses the boundary this leads the high switching speed applications. When we introduce low mobility dopants, hysteresis loop becomes a linear curve. In a yakopcic model, based on the fitting parameter values, the I-V graph is changes. When the threshold voltage $\mathrm{Vp}$ is greater than the maximum input voltage, it acts as a normal resistor instead of memory operation. Ap, An, Vp, Vn plays a vital role in a switching simulated I-V graph [17].

\section{VTEAM MODEL METRICS ANALYSIS}

Quality of models validation is evaluated by its performance against some important model metrics. Different model metrics are analysed for different models [18]. Among a good deal of metrics, accuracy plays a vital role.

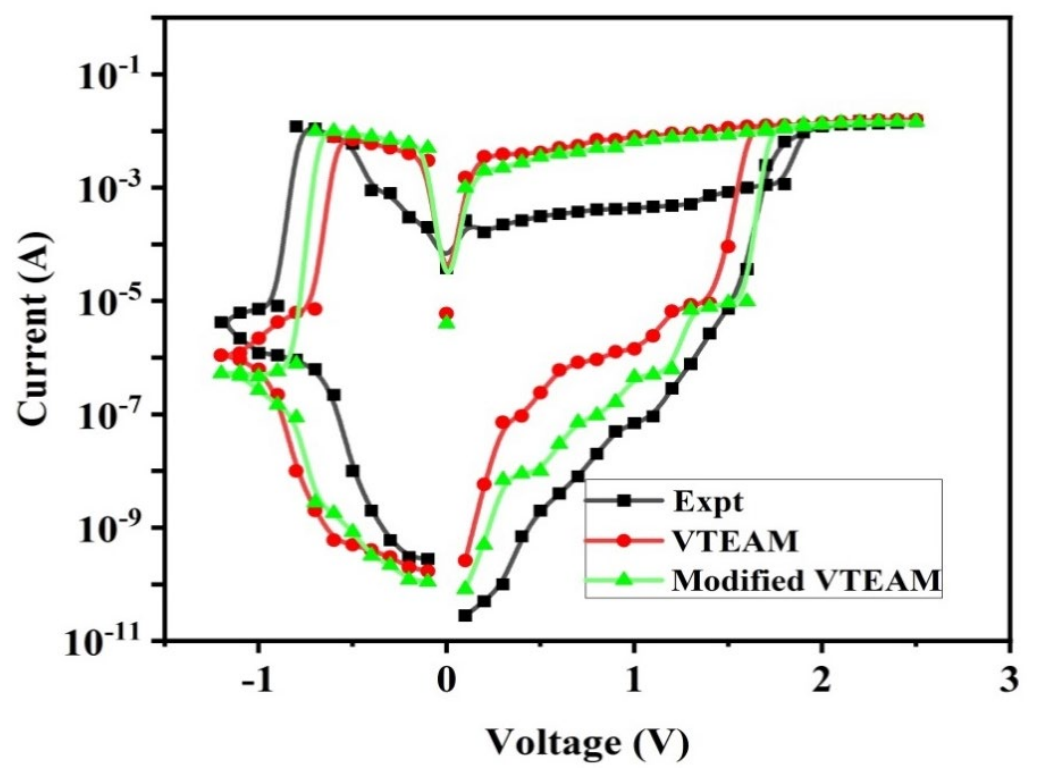

Fig. 5 I-V VTEAM and modified VTEAM models simulated output with experiment result

Accuracy of the VTEAM model is calculated in terms of the mean error of the simulated result with the experimental result, Fig 5 proves that, VTEAM model accuracy is a decent equivalent with the fabricated device results [7].By the further statistical analysis of VTEAM, modified VTEAM models versus the experimental graph, the mean error percentage is calculated as 25.3 and 21.4 respectively. This value is comparable with the IM2NP model [18].

\section{CONCLUSION}

Single-layer nanoscale $\mathrm{Pt} / \mathrm{HfO}_{2} / \mathrm{Pt}$ metal oxide RRAM experimental results are validated with three different appropriate models (non-linear ion drift, Yakopcic and VTEAM) as per the fabricated device structure. Generalised 
model is implemented in a cadence environment with Verilog-A codes according to the showed single memory cell. Simulated results are compared with the experimental results. The comparison table confirms that Modified VTEAM model is exactly fitted with our fabricated single layer switching results. Model metrics of the VTEAM and modified VTEAM models accuracy are analysed in term of its calculated mean error percentage value. This work motivates the researcher to fill the gap between the device levels into next circuit level.

\section{Acknowledgements}

Part of the reported work (fabrication) was carried out at the CeNSE, IISc Bangalore-India under INUP, which has been sponsored by Government of India.

Funding Statement- This research did not receive any specific grant from funding agencies in the public, commercial, or not-for-profit sectors.

Conflict of interest - Nil, (The authors have no conflicts of interest to declare that are relevant to the content of this article)

Availability of data and material - Not applicable

Code availability- Not applicable

Authors' contributions

Author 1: Napolean A

Conceived and designed the analysis, collected the data, contributed data or analysis tools, performed the analysis, wrote the paper.

Author 2: Sivamangai NM

Other works (Suggestion)

Author 3: Nithya N \& Naveenkumar R and

Other works (Editing work)

Ethics approval -Not applicable

Consent to participate -Not applicable

Consent for publication -Not applicable

\section{References}

[1] K. M. Kim, D. S. Jeong, and C. S. Hwang, "Nanofilamentary resistive switching in binary oxide system; a review on the present status and outlook.” Nanotechnology, vol. 22, no.25, p. 254002, Jun. 2011. DOI: $\quad 10.1088 / 0957-4484 / 22 / 25 / 254002$ 
[2] A. Fantini, G. Gorine, R. Degraeve, L. Goux, C. Y. Chen, A. Redolfi, S. Clima, A. Cabrini, G. Torelli, and M. Jurczak, "Intrinsic Program Instability in HfO2 RRAM and consequences on program algorithms.” in Electron Devices Meeting, pp: 7.5.1-7.5.4, Feb. 2016. DOI: 10.1109/IEDM.2015.7409648

[3] H. Y. Jeong, Y. I. Kim, J. Y. Lee, and S. Y. Choi, “A low-temperature-grown TiO2-based device for the flexible stacked RRAM application." Nanotechnology, vol. 21, no.11, p. 115203, Feb, 2010. DOI: 10.1088/0957- 4484/21/11/115203

[4] Wei Zhang, Ji-Zhou Kong, Zheng-Yi Cao, Ai-Dong Li, Lai-Guo Wang, Lin Zhu, Xin Li, Yan-Qiang Cao and Di Wu "Bipolar Resistive Switching Characteristics of HfO2/TiO2/HfO2 Trilayer-Structure RRAM Devices on Pt and TiN-Coated Substrates Fabricated by Atomic Layer Deposition” Nanoscale Research Letters (2017) 12:393.

[5] Kai-Chi Chuang et al (2017) Effects of post-metal annealing on the electrical characteristics of HfOx-based resistive switching memory devices. Japanese Journal of Applied Physics 56:1-31.

[6] Debashis Panda, Paritosh Piyush Sahu and Tseung Yuen Tseng "A Collective Study on Modeling and Simulation of Resistive Random Access Memory" Nanoscale Research Letters (2018) 13:8

[7] A. Napolean, N.M.Sivamagai, R.Naveenkumar, N.Nithya, "Electroforming Atmospheric Temperature and Annealing Effects on Pt/HfO2/TiO2/HfO2/Pt Resistive Random Access Memory Cell”, Silicon, 25 march 2021, https://doi.org/10.1007/s12633-021-01074-8

[8] Strukov, D. B., \& Williams, R. S. (2008). Exponential ionic drift: fast switching and low volatility of thin-film memristors. Applied Physics A, 94(3), 515-519. doi:10.1007/s00339-008-4975-3

[9] Yang JJ, Pickett MD, Li X, Ohlberg DAA, Stewart DR, Williams RS (2008) Memristive switching mechanism for metal/oxide/metal nanodevices. Nat Nanotechnol 3:429-433 Available from: www.nature.com/articles/nnano 2008.160

[10] Strukov DB, Snider GS, Stewart DR, Williams RS (2008) The missing memristor found. Nature 453:80-83 Available from: https://www.nature.com/articles/nature06932

[11] Nagel L (1975) SPICE2, a computer progrem to simulate semiconductor circuit. ERL MemolERL-M 520 Univ. Calif. Univeristy of California Berkeley, Electronics Research Laboratory Available from: https://www2.eecs.berkeley. edu/Pubs/TechRpts/1975/9602.html

[12] Quarles T, Newton AR, Pederson DO, Sangiovanni-Vincentelli A (1994) SPICE 3 version 3F5 user's manual. Dep. Electr. Eng. Comput. Sci. Univ. California, Berkeley, pp 395-404 Available from: https://newton.ex.ac.uk/teaching/ CDHW/Electronics2/userguide.

[13] Yakopcic C, Taha TM, Subramanyam G, Pino RE, Rogers S (2011) A memristor device model. IEEE Electron Device Lett. 32:1436-1438 Available from https://doi.org/10.1109/LED.2011.2163292

[14] Yakopcic C, Taha TM, Subramanyam G, Pino RE (2013) Generalized memristive device SPICE model and its application in circuit design. IEEE Trans Comput Deisgn Integr Circuits Syst 32:1201-1214 Available from: https://doi.org/10.1109/TCAD.2013.2252057

[15] Pickett MD, Strukov DB, Borghetti JL, Yang JJ, Snider GS, Stewart DR et al (2009) Switching dynamics in titanium dioxide memristive devices. J Appl Phys 106:1-6 Available from: https://doi.org/10.1063/1.3236506

[16] Williams RS, Pickett MD, Strachan JP (2013) Physics-based memristor models, Proc.-IEEE Int. Symp. Circuits Syst. pp 217-220 Available from: https://doi.org/10.1109/ ISCAS.2013.65718

[17] Yakopcic C, Taha TM, Subramanyam G, Pino RE (2013) Generalized memristive device SPICE model and its application in circuit design. IEEE Trans Comput Deisgn Integr Circuits Syst 32:1201-1214 Available from: https://doi.org/10.1109/TCAD.2013.2252057.

[18] Basma Hajri, Hassen Aziza, Mohammad M. Mansour, and Ali Chehab, Identifier 10.1109/ACCESS.2017.Doi Number RRAM Device Models: A Comparative Analysis with Experimental Validation, IEEE Access · November 2019, DOI: 10.1109/ACCESS.2019.2954753 